\title{
Association of Delta-Aminolevulinic Acid Dehydratase Gene Variant with Serum Level of Alanine Aminotransferase
}

\author{
Ashraf Mohamadkhani ${ }^{1,}{ }^{,}$, Masoumeh Pourasgari ${ }^{2}$, Mehrnaz Saveh ${ }^{3}$, Hamid-reza Fazli ${ }^{1}$, Parisa \\ Shahnazari ${ }^{4}$ and Hossein Poustchi ${ }^{1}$ \\ ${ }^{1}$ Liver and Pancreatobiliary Diseases Research Center, Digestive Disease Research Institute, Shariati Hospital, Tehran University of Medical Sciences, Tehran, Iran \\ ${ }^{2}$ Cell and Molecular Biology Department, Faculty of Biological Sciences, Kharazmi University, Tehran, Iran \\ ${ }^{3}$ Department of Biology, North Tehran Branch, Islamic Azad University, Tehran, Iran \\ ${ }^{4}$ Department of Bioinformatics, University of Tehran, Tehran, Iran \\ "Corresponding author: Liver and Pancreatobiliary Diseases Research Center, Digestive Disease Research Institute, Shariati Hospital, Tehran University of Medical Sciences, \\ Tehran, Iran. Email: mohamadkhani.ashraf@gmail.com
}

Received 2019 May 29; Revised 2019 July 15; Accepted 2019 August 14.

\begin{abstract}
Background: The enzyme ALAD (delta-aminolevulinic acid dehydratase), encoded by the ALAD gene, catalyzes the synthesis of porphobilinogen. The $177 \mathrm{G}>\mathrm{C}$ ( $\mathrm{rs1800435)}$ ) polymorphism participates in the hereditary deficiency of porphobilinogen synthase to cause acute lead poisoning.

Objectives: The current study aimed at investigating the allelic frequency of the enzyme ALAD in patients with non-alcoholic fatty liver disease (NAFLD) compared to the control group.

Methods: The fatty liver index (FLI) algorithm was used to diagnose NAFLD in participants of a prospective cohort of the Digestive Diseases Research Institute (DDRI). The ALAD genotypes, ALAD1 and ALAD2, were identified in 100 patients with NAFLD and 200 healthy controls using polymerase chain reaction restriction fragment length polymorphism (PCR-RFLP).

Results: The C-allele of ALAD rs1800435 frequency was 5.5\% in the group of patients with NAFLD compared to 3.3\% in the control group, without significant differences $(\mathrm{P}=0.37)$; however, alleles were in the Hardy-Weinberg equilibrium $(\mathrm{P}>0.05)$. Serum ALT level was considerably higher in the ALAD2 carriers group than in the ones not carrying ALAD2 (29.4 \pm 13.9 vs. 19.4 $\pm 10.1, \mathrm{P}=0.041)$. Nonetheless, each C-allele increased the serum ALT level by $1.24 \mathrm{IU} / \mathrm{L}(95 \%$ confidence interval: $0.22-2.67 ; \mathrm{P}=0.04)$.

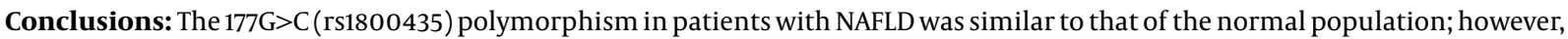
it can be considered as a risk factor for serum ALT level increase.
\end{abstract}

Keywords: Non-Alcoholic Fatty Liver Disease, Aminolevulinic Acid Dehydratase, rs1800435, Alanine Aminotransferase

\section{Background}

Non-alcoholic fatty liver disease (NAFLD) is the most common cause of chronic liver disease, from simple hepatic steatosis to steatohepatitis, which may progress to cirrhosis and eventually to hepatocellular carcinoma. NAFLD is a problem of high morbidity and mortality related to the liver, and also an increase in mortality due to cardiovascular disease (CVD) and cancer (1). Serum alanine aminotransferase (ALT) is a sensitive biomarker of hepatic injury commonly used to screen and detect abnormal liver function and estimate levels of abnormality. The increase in serum ALT level is more closely related to fat accumulation in the liver and reveals histological progression of the liver. Higher serum ALT level accompanied by echogenic liver ultrasonography, in the absence of any identified cause of liver disease, suggests the diagnosis of $\operatorname{NAFLD}(2,3)$.
Despite well-known risk factors for NAFLD such as genetic components and life style, the underlying mechanism of fatty liver is unclear $(2,4,5)$. However, environmental influences such as car engine exhaust particles, metals, and various polychlorinated elements are significant causes of NAFLD progression (6-8). The effect of exposure to air pollutants on the onset of diseases is widely accepted, and reveals a difference in the composition of fatty acids in the liver and adipose tissue that consequently has negative effects on health by increasing the risk of cardiovascular disease, systemic and immune inflammation, and symptoms of depression (9-11). The harmful impact of air pollutants is involved in the pathogenesis of fatty liver from oxidative stress and insulin resistance leading to increased levels of aminotransferase (12).

Delta-aminolevulinic acid dehydratase (ALAD) is a cy- 
tosolic sulfhydryl enzyme strongly inhibited by lead airborne particulates and generally attributed to the pathogenesis of lead poisoning $(13,14)$. Human ALAD is a polymorphic enzyme, encoded by the ALAD gene on chromosome 9q34 and involved in the synthesis of heme by converting aminolevulinate (ALA) to porphobilinogen (PBG). The common variant $177 \mathrm{G}>\mathrm{C}$ ( $\mathrm{rs1800435)}$ in the exon 4 of $A L A D$, which substitutes asparagine with lysine on residue 59, produces two codominant alleles ALAD1 and ALAD2 in three genotypes of ALAD 1-1, ALAD 1-2, and ALAD 2-2 (13, 15). It is shown that carriers of the ALAD2 (C allele) are prone to exhaust particles to have higher blood lead concentrations than the frequent ALAD1 (G allele). The electronegative properties of the ALAD2 enzyme increase its affinity for lead $(16,17)$.

\section{Objectives}

Atmospheric pollution as a major concern in urban environments affects patients with NAFLD; therefore, the current study aimed at investigating the frequency of $A L A D$ genotypes, the enzyme related to air pollution that increases the sensitivity to lead poisoning, in patients with NAFLD compared to healthy individuals and the association of the ALAD rs1800435 polymorphism with serum ALT level.

\section{Methods}

\subsection{Study Subjects}

A total of 300 subjects (179 males and 121 females) in a prospective cohort at a referral clinic affiliated to Tehran University of Medical Sciences were enrolled in the current study. The current nested case-control study was conducted on 100 patients with NAFLD and 200 subjects with normal ALT levels ( $<40 \mathrm{U} / \mathrm{L}$ in males, $<34 \mathrm{U} / \mathrm{L}$ in females) as a control group selected consecutively. The fatty liver index (FLI) algorithm was used to diagnose NAFLD according to the formula published by Huang et al. (18). Selection of ALT threshold values was based on previous studies to estimate the upper health limits in healthy blood donors (3). The study included subjects without a history of alcohol abuse, autoimmune hepatitis, use of hepatotoxic drugs, evidence of viral liver disease, tumors, cholestasis, or other metabolic diseases of the liver. Venous blood samples after a 12-hour overnight fasting were collected from all participants. Demographic data were obtained and the biochemical parameters for each subject were tested using available standardized methods (19). The study protocol was in accordance with the Declaration of Helsinki and was approved by the Ethics Committee of the Digestive Disease
Research Institute (DDRI), Shariati Hospital, Tehran University of Medical Sciences (TUMS) (ethical code: 416/780). Written informed consent was obtained from all subjects.

\subsection{Genotyping for the ALAD Polymorphism}

Genomic DNAs were extracted from the blood samples using the Gentra Puregene kit (Qiagen, Alameda, CA, USA) according to the manufacturer's recommendations. To identify the two variants of $A L A D$, the polymerase chain reaction restriction fragment length polymorphism (PCR-RFLP) with specific primers to detect MspI restriction sites (C/CG G) was performed as described previously (15). The primers used in the ALAD genotyping were: 5'-AGACAGACATTAGCTCAGTA-3' and 5'GGCAAAGACCACGTCCATTC-3' in amplification of a 916-basepair (bp) sequence. PCR products were digested with the MspI restriction enzyme to produce dissimilar fragments that lead to specific genotypes. The fragmented products were then analyzed on the agarose gel. The wild type ALAD1-1, homozygous variants ALAD 2-2 and ALAD 1-2 heterozygous were defined by fragments of 582, 511, and 582 $\mathrm{bp}$ in addition to $511 \mathrm{bp}$, accordingly. The protocol and the condition of the PCR were as previously described (15).

\subsection{Laboratory Measurements}

Serum insulin was measured by ELISA (the enzymelinked immunosorbent assay) technique (Diesse Company, Italy). Lipid profiles, including total cholesterol (TC), triglycerides (TG), high-density lipoprotein-cholesterol (HDL-c), liver enzymes, and fasting plasma glucose were tested using an autoanalyzer (Cobas c 702, Roche; Shanghai, China). Platelets were counted using Sysmex kx-21.

\subsection{Statistical Analysis}

Analysis of variance (ANOVA) was used to compare continuous variables. Chi-square test was used to compare categorical group variables and also determine if the genotype distribution was in the Hardy-Weinberg equilibrium. Logistic regression analysis was used to calculate the odds ratios of the variants for ALT level. SPSS version 15.0 (SPSS, Chicago, IL, USA) was used to analyze data and $\mathrm{P}<0.05$ was considered significant.

\section{Results}

\subsection{Clinical Features of the Study Population}

The general characteristics of the NAFLD and nonNAFLD groups are shown in Table 1. The number of females and males, mean age, and body mass index (BMI) in the NAFLD group were respectively 42 and 58 subjects, $41.1 \pm$ 


\begin{tabular}{|c|c|c|c|}
\hline Characteristics & $\begin{array}{c}\text { Patients with } \\
\text { NAFLD }(N=100)\end{array}$ & $\begin{array}{c}\text { Controls }(\mathbf{N}= \\
200)\end{array}$ & P Value \\
\hline Age, $y$ & $42.3(11.9)$ & $41.1(14.2)$ & 0.48 \\
\hline Gender, female/male & $42 / 58$ & $79 / 121$ & 0.70 \\
\hline BMI, $\mathrm{kg} / \mathrm{m}^{2}$ & $24.7(5.4)$ & $23.98(4.6)$ & 0.18 \\
\hline Platelet $\times 10^{9} / \mathrm{L}$ & $304.42(89.01)$ & $293.70(77.42)$ & 0.25 \\
\hline ALT, IU/L & $42.7(8.3)$ & $16.9(5.7)$ & 0.000 \\
\hline ALP, IU/L & $192.5(55.6)$ & $169.6(66.8)$ & 0.03 \\
\hline Cholesterol, mg/dL & $188.4(40.7)$ & $165.0(33.6)$ & 0.000 \\
\hline HDL, mg/dL & $45.4(10.1)$ & $47.6(10.0)$ & 0.075 \\
\hline TG, mg/dL & $198.3(153.5)$ & $112.0(52.7)$ & 0.000 \\
\hline FBS, $\mathrm{mg} / \mathrm{dL}$ & $98.4(18.6)$ & $85.6(19.4)$ & 0.092 \\
\hline Insulin, IU/mL & $9.50(5.56)$ & $7.86(6.24)$ & 0.027 \\
\hline
\end{tabular}

Abbreviations: ALP, alkaline phosphatase; ALT, alanine aminotransferase; FBS, fasting blood sugar; Hb, hemoglobin; HDL, high-density lipoprotein; TG triglyceride.

${ }^{\mathrm{a}}$ Values are expressed as mean (SD)

14.2 years, and $24.7 \pm 5.4 \mathrm{~kg} / \mathrm{m}^{2}$ compared to 79 and $121 \mathrm{sub}-$ jects, $11.44 \pm 2.99$ years, and $23.98 \pm 4.6 \mathrm{~kg} / \mathrm{m}^{2}$, which were not significantly different. The mean ALT, ALP, TG, cholesterol, and serum insulin levels were significantly higher in the NAFLD group than in the non-NAFLD group $(\mathrm{P}<0.001)$.

\subsection{ALAD Genotypes and Allele Distribution}

According to the findings, $10 \%$ of the patients with NAFLD were ALAD2 carriers with both ALAD 2-2 (1\%) and ALAD 1-2 (9\%) genotypes. This rate was $6.5 \%$ in the control group, all with ALAD 1-2 genotype, without significant differences $(\mathrm{P}>0.09)$.

The frequency of the C-allele of ALAD rs1800435 was $5.5 \%$ in patients and $3.3 \%$ in controls, with a borderline difference $(P=0.07)$; however, both the $\mathrm{G}$ - and the $\mathrm{C}$-alleles were in the Hardy-Weinberg equilibrium $(\mathrm{P}>0.05)$. Table 2 revealed the allelic frequency of the ALAD rs1800435 polymorphism and the genotype distribution between the patients and controls.

To assess whether $177 \mathrm{G}>\mathrm{C}$ (rs1800435) polymorphism influences clinical parameters; the mean \pm standard deviation (SD) of the variables was compared between the carriers and non-carriers of $A L A D$ rs1800435. As shown in Table 3, the serum ALT level was considerably higher in the ALAD2 carriers than in non-carriers of ALAD2 (29.4 \pm 13.9 vs. 19.4 $\pm 10.1, \mathrm{P}=0.041)$. However, no significant differences were observed in other experimental features and demographic data among the study groups $(\mathrm{P}>0.05)$. Using the linear regression adjusted for age, BMI, and gender, a significant association was observed between the ALAD2 genotype and the ALT level. For ALAD rs1800435, each C-allele increased the ALT level by $1.24 \mathrm{IU} / \mathrm{L}$ (95\% confidence interval (CI): 0.22 - 2.67; $\mathrm{P}=0.04)$.

\section{Discussion}

The effects of environmental factors such as air pollution on the incidence of NAFLD along with an increase in liver enzyme levels and consequent steatosis were previously reported $(6,9,20)$. Exposure to diesel exhaust particles in diabetic obese mice is positively associated with NAFLD, and mortality due to diabetes mellitus is probably through increased oxidative stress (10). This situation is important to explore the contribution of variants in $A L A D$ gene related to lead toxicity in common diseases such as NAFLD. Although the ALAD rs1800435 polymorphism has important effects on the susceptibility to toxicity of lead particle, information on the distribution of $A L A D$ gene polymorphism in NAFLD subjects is not provided. Furthermore, there was no evidence to demonstrate the distribution of genetic variants of the $A L A D$ genotypes in the Iranian population.

The current study results showed that the distribution of $A L A D$ genotypes in patients with NAFLD compared to healthy subjects had no significant differences and also allelic variations of $A L A D$ locus showed similar frequencies in both study groups. Previous studies confirmed that ALAD2 carriers are generally more likely to have a high blood lead level (14, 21, 22); however, blood lead was not measured in the current study. Though, carriers of ALAD 177G $>C$ variants in the current study showed an increase in serum ALT; therefore, it could be evidence for an association between ALAD genotypes and predisposition to NAFLD. The serum ALT level is a sensitive indicator and one of the key tests to recognize, screen, and follow-up the patients with hepatitis. The significance of ALT activity as an index of liver damage was examined in previous studies $(23,24)$. Furthermore, the allelic frequency of $A L A D$ in the current study was very similar to that of previously reported in Caucasian and Asian populations with distribution of $92 \%$ for ALAD 1-1 and $8 \%$ for ALAD 1-2 $(22,25)$. Therefore, the current study results suggested a consistency in the distribution of ALAD $177 \mathrm{G}>\mathrm{C}$ (rs1800435) variants in the Iranian population.

\subsection{Conclusions}

In conclusion, although there was no difference in the distribution of $A L A D$ genotypes among the patient groups with controls; however, ALAD2 carriers had a higher serum ALT level. Air pollution has the most important effects on human health, causing numerous diseases and leading to 


\begin{tabular}{|c|c|c|c|c|c|}
\hline \multirow{2}{*}{$\begin{array}{l}\text { Variants }^{\mathrm{a}} \\
\text { ALAD 177G }>\mathrm{C}(\text { (rs1800435 })\end{array}$} & \multicolumn{2}{|c|}{ Patients with NAFLD $(\mathrm{N}=100)$} & \multicolumn{2}{|c|}{ Controls $(\mathrm{N}=\mathbf{2 0 0})$} & \multirow{2}{*}{$\begin{array}{c}\text { P Value } \\
0.07\end{array}$} \\
\hline & & & & & \\
\hline Genotype & $\mathbf{N}$ & $\mathbf{G F}(\%)$ & $\mathbf{N}$ & $\mathbf{G F}(\%)$ & \\
\hline GG & 90 & 90 & 187 & 93.5 & \\
\hline CG & 9 & 9 & 13 & 6.5 & \\
\hline Allele & $\mathbf{N}$ & $\mathbf{A F}(\%)$ & $\mathbf{N}$ & $\mathbf{A F}(\%)$ & \\
\hline G(ancestral) & 189 & 94.5 & 387 & 96.7 & \\
\hline $\mathrm{C}$ (minor) & 11 & 5.5 & 13 & 3.3 & \\
\hline
\end{tabular}

${ }^{\mathrm{a}}$ Genotypic and allelic frequencies are shown as absolute and percentage data.

\begin{tabular}{|c|c|c|c|}
\hline Characteristic & $\begin{array}{c}\text { ALAD2 } \\
\text { Carriers }(\mathrm{N}= \\
100)\end{array}$ & $\begin{array}{c}\text { ALAD1 } \\
\text { Carriers }(\mathrm{N}= \\
\mathbf{2 0 0})\end{array}$ & PValue \\
\hline Age, $y$ & $39.6(15.4)$ & $41.7(13.2)$ & 0.53 \\
\hline Gender, female/male & $8 / 15$ & $113 / 164$ & 0.66 \\
\hline BMI, $\mathrm{kg} / \mathrm{m}^{2}$ & $23.5(4.8)$ & $24.3(4.9)$ & 0.49 \\
\hline Platelet $\times 10^{9} / \mathrm{L}$ & $311.12(91.2)$ & $291.62(85.9)$ & 0.19 \\
\hline ALT, IU/L & $29.4(13.9)$ & $19.4(10.1)$ & 0.041 \\
\hline ALP, IU/L & $207.0(104.3)$ & $147.7(59.2)$ & 0.15 \\
\hline Cholesterol, mg/dL & $174.1(50.3)$ & $172.7(36.6)$ & 0.89 \\
\hline HDL, mg/dL & $46.2(9.1)$ & $46.9(10.2)$ & 0.71 \\
\hline TG, mg/dL & $146.9(125.4)$ & $140.3(104.8)$ & 0.80 \\
\hline FBS, $\mathrm{mg} / \mathrm{dL}$ & $90.0(26.0)$ & $86.2(18.5)$ & 0.50 \\
\hline Insulin, IU/mL & $7.64(4.33)$ & $8.47(6.19)$ & 0.40 \\
\hline
\end{tabular}

${ }^{\mathrm{a}}$ Values are expressed as mean (SD).

high morbidity and mortality, especially in the developing countries. Therefore, with reference to the hypothesis that air pollution influences the development of NAFLD, as an important economic burden and health problem, it must be assessed by measuring the level of lead in blood and through mechanisms related to systemic oxidative stress.

\section{Footnotes}

Authors' Contribution: Mehrnaz Saveh and Masoumeh Pourasgari perform laboratory tasks; Parisa Shahnazari and Hamid-reza Fazli were responsible for the statistical calculations. Ashraf Mohamadkhani planned and wrote the manuscript. Hossein Poustchi accepted for patient management and samples.

Conflict of Interests: Authors declared no conflict of interest.
Ethical Approval: The study protocol was carried out in accordance with the Helsinki Declaration and was approved by the Ethics Committee of the Digestive Disease Research Institute (DDRI).

Funding/Support: The study was supported by Digestive Disease Research Institute (DDRI), Shariati Hospital, Tehran University of Medical Sciences (TUMS).

\section{References}

1. Nahandi MZ, Khoshbaten M, Ramazanzadeh E, Abbaszadeh L, Javadrashid R, Shirazi KM, et al. Effect of non-alcoholic fatty liver disease on carotid artery intima-media thickness as a risk factor for atherosclerosis. Gastroenterol Hepatol Bed Bench. 2014;7(1):55-62. [PubMed: 25436098]. [PubMed Central: PMC4017554].

2. Alwahsh SM, Gebhardt R. Dietary fructose as a risk factor for nonalcoholic fatty liver disease (NAFLD). Arch Toxicol. 2017;91(4):1545-63. doi: 10.1007/s00204-016-1892-7. [PubMed: 27995280].

3. Mohamadnejad M, Pourshams A, Malekzadeh R, Mohamadkhani A, Rajabiani A, Asgari AA, et al. Healthy ranges of serum alanine aminotransferase levels in Iranian blood donors. World J Gastroenterol. 2003;9(10):2322-4. doi: 10.3748/wjg.v9.110.2322. [PubMed: 14562401]. [PubMed Central: PMC4656486].

4. Alsabaani AA, Mahfouz AA, Awadalla NJ, Musa MJ, Al Humayed SM. Non-alcoholic fatty liver disease among type-2 diabetes mellitus patients in Abha City, South Western Saudi Arabia. Int J Environ Res Public Health. 2018;15(11). doi: 10.3390/ijerph15112521. [PubMed: 30423871]. [PubMed Central: PMC6266142].

5. Erfanian S, Moradzadeh M, Solhjoo K, Jahromi AS. Data describing the association between rs266729 polymorphism inadiponectin promoter gene and Type 2 Diabetes Mellitus. Data Brief. 2016;9:1138-40. doi: 10.1016/j.dib.2016.11.040. [PubMed: 27942572]. [PubMed Central: PMC5133470].

6. Zheng Z, Xu X, Zhang X, Wang A, Zhang C, Huttemann M, et al. Exposure to ambient particulate matter induces a NASH-like phenotype and impairs hepatic glucose metabolism in an animal model J Hepatol. 2013;58(1):148-54. doi: 10.1016/j.jhep.2012.08.009. [PubMed: 22902548]. [PubMed Central: PMC3527686].

7. Ghorani-Azam A, Riahi-Zanjani B, Balali-Mood M. Effects of air pollution on human health and practical measures for prevention in Iran. J Res Med Sci. 2016;21:65. doi: 10.4103/1735-1995.189646. [PubMed: 27904610]. [PubMed Central: PMC5122104].

8. Hatami B, Ashtari S, Sharifian A, Rahmani Seraji H, Khalili E, Hatami Y, et al. Changing the cause of liver cirrhosis from hepatitis $B$ 
virus to fatty liver in Iranian patients. Gastroenterol Hepatol Bed Bench. 2017;10(Suppl1):S20-6. [PubMed: 29511467]. [PubMed Central: PMC5838176].

9. Hong YC, Lee JT, Kim H, Kwon HJ. Air pollution: A new risk factor in ischemic stroke mortality. Stroke. 2002;33(9):2165-9. doi: 10.1161/01.str.0000026865.52610.5b. [PubMed: 12215581].

10. Tomaru M, Takano H, Inoue K, Yanagisawa R, Osakabe N, Yasuda A, et al. Pulmonary exposure to diesel exhaust particles enhances fatty change of the liver in obese diabetic mice. Int J Mol Med.2007;19(1):1722. [PubMed: 17143543].

11. Bucher S, Tete A, Podechard N, Liamin M, Le Guillou D, Chevanne M, et al. Co-exposure to benzo[a]pyrene and ethanol induces a pathological progression of liver steatosis in vitro and in vivo. Sci Rep. 2018;8(1):5963. doi: 10.1038/s41598-018-24403-1. [PubMed: 29654281]. [PubMed Central: PMC5899096].

12. Perez M, Gonzales L, Olarte R, Rodriguez NI, Tabares M, Salazar JP, et al. Nonalcoholic fatty liver disease is associated with insulin resistance in a young Hispanic population. Prev Med. 2011;52(2):174-7. doi 10.1016/j.ypmed.2010.11.021. [PubMed: 21147154].

13. Suzen HS, Duydu Y, Aydin A. Molecular analysis of deltaaminolevulinic acid dehydratase (ALAD) gene polymorphism in a Turkish population. Biochem Genet. 2004;42(11-12):461-7. [PubMed 15587989].

14. Kayaalti Z, Sert S, Kaya-Akyuzlu D, Soylemez E, Soylemezoglu T. Association between delta-aminolevulinic acid dehydratase polymorphism and placental lead levels. Environ Toxicol Pharmacol.2016;41:147-51. doi 10.1016/j.etap.2015.11.017. [PubMed: 26701682].

15. Montenegro MF, Barbosa F Jr, Sandrim VC, Gerlach RF, Tanus-Santos JE. Ethnicity affects the distribution of delta-aminolevulinic acid dehydratase (ALAD) genetic variants. Clin Chim Acta. 2006;367(1-2):192-5 doi: 10.1016/j.cca.2005.12.009. [PubMed: 16445899].

16. Montenegro MF, Barbosa F Jr, Sandrim VC, Gerlach RF, Tanus-Santos JE. A polymorphism in the delta-aminolevulinic acid dehydratase gene modifies plasma/whole blood lead ratio. Arch Toxicol. 2006;80(7):3948. doi: 10.1007/s00204-005-0056-y. [PubMed:16341691].

17. Fujihara J, Agusa T, Yasuda T, Soejima M, Kato H, Panduro A, et al. Ethnic variation in genotype frequencies of delta-aminolevulinic acid dehydratase (ALAD). Toxicol Lett. 2009;191(2-3):236-9. doi: 10.1016/j.toxlet.2009.09.005. [PubMed: 19766174].

18. Huang X, Xu M, Chen Y, Peng K, Huang Y, Wang P, et al. Validation of the fatty liver index for nonalcoholic fatty liver disease in middleaged and elderly Chinese. Medicine (Baltimore). 2015;94(40). e1682. doi: 10.1097/MD.0000000000001682. [PubMed: 26448014]. [PubMed Central: PMC4616754]

19. Mohamadkhani A, Poustchi H. Repository of human blood derivative biospecimens in biobank: Technical implications. Middle East J Dig Dis. 2015;7(2):61-8. [PubMed: 26106464]. [PubMed Central: PMC4430793].

20. Hiyoshi M, Uemura H, Arisawa K, Nakamoto M, Hishida A, Okada R, et al. Association between the catechol-O-methyltransferase (rs4680: Val158Met) polymorphism and serum alanine aminotransferase activity. Gene. 2012;496(2):97-102. doi: 10.1016/j.gene.2012.01.015. [PubMed: 22293393].

21. La-Llave-Leon O, Mendez-Hernandez EM, Castellanos-Juarez FX, Esquivel-Rodriguez E, Vazquez-Alaniz F, Sandoval-Carrillo A, et al. Association between blood lead levels and delta-aminolevulinic acid dehydratase in pregnant women. Int J Environ Res Public Health. 2017;14(4). doi: 10.3390/ijerph14040432. [PubMed: 28420209]. [PubMed Central: PMC5409633].

22. Shen XM, Wu SH, Yan CH, Zhao W, Ao LM, Zhang YW, et al. Deltaaminolevulinate dehydratase polymorphism and blood lead levels in Chinese children. Environ Res. 2001;85(3):185-90. doi: 10.1006/enrs.2000.4230. [PubMed: 11237505].

23. Prati D, Taioli E, Zanella A, Della Torre E, Butelli S, Del Vecchio E, et al. Updated definitions of healthy ranges for serum alanine aminotransferase levels. Ann Intern Med. 2002;137(1):1-10. doi: 10.7326/0003-4819137-1-200207020-00006. [PubMed:12093239].

24. Miyake T, Kumagi T, Hirooka M, Koizumi M, Furukawa S, Ueda T, et al. Metabolic markers and ALT cutoff level for diagnosing nonalcoholic fatty liver disease: A community-based cross-sectional study. J Gastroenterol. 2012;47(6):696-703. doi: 10.1007/s00535-012-0534-y. [PubMed: 22331365].

25. Ben-Ezzer J, Oelsner H, Szeinberg A. Genetic polymorphism of delta-aminolevulinate dehydrase in several population groups in Israel. Hum Hered. 1987;37(4):229-32. doi: 10.1159/000153708. [PubMed: 3653904]. 